\title{
Median Basilic Vein
}

National Cancer Institute

\section{Source}

National Cancer Institute. Median Basilic Vein. NCI Thesaurus. Code C33070.

A vein between the biceps and pronator radii teres muscles that unites with the common ulnar vein to form the basilic vein within the forearm. 